\title{
Potential Biomedical Applications of Terahertz Technologies
}

\author{
Xiao Yu Peng1*, Fei Chen ${ }^{1}$ and Ben Liang Xie ${ }^{2}$ \\ ${ }^{1}$ Chongqing Institute of Green and Intelligent Technology, Chinese Academy of Sciences, China \\ ${ }^{2}$ School of Physics and Electronic Information, Gannan Normal University, China \\ *Corresponding author: Xiao Yu Peng, Chongqing Institute of Green and Intelligent Technology, Chinese Academy of Sciences, Chong- \\ qing 400714, China.
}

To Cite This Article: Xiao Yu Peng, Potential Biomedical Applications of Terahertz Technologies. 2020 - 10(2). AJBSR.MS.ID.001490.

DOI: 10.34297/AJBSR.2020.10.001490.

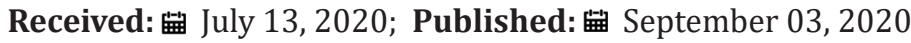

\begin{abstract}
Terahertz (THz) Technologies applied in biomedical science are reviewed in this paper. Current research results indicate that THz technologies would be able to provide not only potential novel detection techniques for cancer diagnostics but also potential novel therapies for cancers and skin disease treatment.
\end{abstract}

\section{Introduction}

Science and technologies based on THz wave, electromagnetic radiation with its frequency ranges from 0.1 to $10 \mathrm{THz}$ and its wavelength ranges from $3 \mathrm{~mm}$ to $30 \mu \mathrm{m}$, have developed rapidly over the last 30 years. Especially following the development of femto-second-laser-based $\mathrm{THz}$ time-domain spectroscopy (THz TDS) and THz imaging techniques1, the field of THz science and technology expanded rapidly. For example, THz-TDS systems have been used not only in the fundamental science such as the investigation of different motion modes of the molecules, lattice vibration of crystals, optimizing materials for new solar cells et al., but also in some real applications such as detection/recognition of chemicals, explosives, drugs, and pharmaceuticals, non-destructive inspection of pharmaceutical tablets and the paint quality of the vehicles. In addition, passive THz imaging technique has been used as a key technology for next generation of airport security scanners. In biomedical science, $\mathrm{THz}$ technologies have also shown their potential applications, including not only potential novel detection techniques for skin tumor diagnostics but also potential novel therapies for skin cancers and skin diseases.

\section{THz spectroscopy and THz Imaging: Potential Novel} Diagnostics

There are three reasons why $\mathrm{THz}$ technologies may be used potentially in biomedical science as new diagnostics: (1) resonances in the $\mathrm{THz}$ frequency range in biomacromolecules, being the basic of the $\mathrm{THz}$ detection; (2) low possibility of damage on cells or tissues due to the low energy photons (1THz4.1meV), suitable for non-destructive detection on human body; (3) strong absorbance by water in cells or tissues.

A multitude resonance associated with twisting, helix, and liberational modes in biomacromolecules and the weak intermolecular interaction (hydrogen bond or Van der Waals interaction force) between them were predicted theoretically in $\mathrm{THz}$ frequency range and proved by experimental measurements. For example, a multitude resonance absorption peaks have been observed in five typical nucleobases of DNA/RNA [1,2] and amino acids [3] in the entire $\mathrm{THz}$ range. The $\mathrm{THz}$ molecular resonances cab be quantified to identify the types of cancer cells with a certain degree of DNA methylation, revealing these resonance absorption 
peaks could be utilized for potential early diagnosis of cancers at the molecular level [4].

These results indicate that the characteristic resonance absorption peaks could be used as "fingerprints" for recognizing biomacromolecules, analysing the small change of the structure and components of these molecules or cells, providing new strategy for medical diagnostics. In vivo detection, big challenge is the strong absorbance by extra/intracellular water, resulting in a smooth broad frequency dependent absorption and very low Signal to Noise Ratio (SNR). To increase the SNR, the disadvantage of bulk water for solution phase measurement can be removed by freezing the sample or utilizing THz ATR silicon prism [5]. For human tissue (such as skin) detection in vivo, ATR-prism-combined technique [6] is a better choice.

Another promising application of $\mathrm{THz}$ technology for diagnostics is $\mathrm{THz}$ imaging, a non-invasive and label-free tool, motivated by strong absorbance by water and different absorbance between normal tissues and tumours due to the sensitivity of $\mathrm{THz}$ absorption to water content and tissue structure, and the degree of hydration of tissue could be used as an indicator of disease state. For example, THz dynamic imaging based on THz-TDS can be used to trace the skin drug absorption [7]. THz imaging showed its capability to margin the skin cancer $[8]$ and breast cancer $[9,10]$ from around normal tissues prior to surgery. Numerous groups have demonstrated medical applications of far/near field $\mathrm{THz}$ imaging in colon cancer, oral cancer, gliomas, burn tissue, wound tissue, tooth healthcare, corneal hydration, and so on, both in vivo and ex vivo.

The prototype intra-operative surgical probe has been designed for use during breast cancer surgery. This endoscopy like devices would be suitable for in vivo cancer screening. Besides, a $\mathrm{THz}$ molecular imaging technique [11] with contrast-agent-like nanoparticle probes was proposed, shown its potential applications such as cancer diagnosis, drug delivery monitoring, and stem cell tracking.

\section{Biological Effects of THz Radiation: Potential Novel Therapies}

Above mentioned $\mathrm{THz}$ waves for potential clinical diagnostics are known as safe due to their low energy photons without ionization damage. However, with the increase of power/energy of THz radiation, many biological effects on living cells and tissues such as the main thermal effect for continued THz wave or pulsed $\mathrm{THz}$ wave with high repetition rate and the main nonlinear resonance effect for pulsed $\mathrm{THz}$ wave with low repletion rate can be observed routinely. For example, to study the thermal effects of $\mathrm{THz}$ waves, human dermal fibroblasts were exposed to continuous $\mathrm{THz}$ radiation $(2.52 \mathrm{THz}, 84.8 \mathrm{~mW} / \mathrm{cm} 2)$. After tens of minutes irradiated, it was found that cell viability was significantly affected when cellular temperatures increased by $3^{\circ} \mathrm{C}$, and the expression of both heat shock proteins and DNA damage markers tended to increase, suggesting that radiation at $2.52 \mathrm{THz}$ generates predominantly thermal effects in mammalian cells [12]. The non-thermal biological effects of THz waves have received greater attention in past years. For example, intense THz pulse can lead to high expression of some tumor suppressors and down-regulate genes associated with skin cancer and psoriasis [13]. Six-hour irradiation with a broadband $\mathrm{THz}$ source results in specific change in gene expression and also the biological functions in human eye cells [14]. Ten minutes exposure to intense $\mathrm{THz}$ pulses leads to a significant induction of H2AX phosphorylation, indicating that $\mathrm{THz}$ pulse irradiation may cause DNA damage in exposed skin tissue [15]. Besides, intense $\mathrm{THz}$ pulse can cause injury to the morphology of living cells and affect the permeability of cell membrane [16], lead to apoptosis or even necrosis and damage to biological tissues [17].

Above mention biological effects of $\mathrm{THz}$ radiation remind us that it is necessary to consider the negative effects of high power/energy $\mathrm{THz}$ radiation on human body and establish some protection standards when people use these strong THz sources. On the other hand, these biological effects indicate that enough dose of irradiation of the high power/energy THz sources can provide potential novel therapies for cancers and skin diseases treatment, which have been proved preliminarily by an experiment on tumor ablation using a gyrotron $\mathrm{THz}$ source [18].

\section{Conclusion}

THz technologies based on THz spectroscopy and THz imaging have been used in scientific research in biomedical science. These technologies show potential novel diagnostics for cancer detection or other skin diseases. With the increase of power/energy of $\mathrm{THz}$ radiation, many biological effects including thermal effect and nonlinear resonance effects can be observed routinely. Even though $\mathrm{THz}$ technologies applied in biomedical science are still in their infancy and there are still some challenges, however, a great deal of current research results of these technologies have shown that they would provide not only potential novel skin cancer diagnostics but also potential novel therapies for skin cancers and skin diseases.

\section{Acknowledgment}

We acknowledge the support from the National Key R\&D Program of China (Grant No. 2017YFA0701002).

\section{References}

1. Mickan SP, Zhang XC (2003) T-Ray Sensing and Imaging. International Journal of High-Speed Electronics and Systems 13(2): 601-676.

2. Yu M, Yan S, Sun YQ, Sheng W, Tang F, et al. (2019) Characterization of Nucleobases in Broadband Terahertz Spectra from 0.5 to $10 \mathrm{THz}$ with the Air-Biased-Coherent-Detection Technique. Sensors 19(5): 1148. 
3. Yi W, et al. (2017) Instrum Sci Tech 45: 423.

4. Cheon H, Hee jin Y, Sang Hun L, Kim YA, Joo Hiuk S (2016) Terahertz molecular resonance of cancer DNA. Sci Rep 6: 37103.

5. Zou Y, et al. (2018) Biomed Opt Express 9: 14.

6. Chen X, Pickwell MacPherson E (2019) A Sensitive and Versatile Thickness Determination Method Based on Non-Inflection Terahertz Property Fitting. Sensors 19(19): 4118.

7. Hu BB, Nuss MC (1995) Imaging with terahertz waves. Opt Lett 20(16): 1716-1718.

8. Wallace VP, Fitzgerald AJ, Shankar S, Flanagan N, Pye R, et al. (2004) Terahertz pulsed imaging of basal cell carcinoma ex vivo and in vivo. $\mathrm{Br}$ J Dermatol 151(2): 424-432.

9. Fitzgerald AJ, Wallace VP, Jimenez Linan M, Bobrow L, Pye RJ, et al. (2006) Terahertz pulsed imaging of human breast tumors. Radiology 239(2): 533-540

10. El Shenawee M, Nagma V, Tyler B, Keith B (2019) Cancer detection in excised breast tumors using terahertz imaging and spectroscopy. Biomedical Spectroscopy and Imaging 8(1-2): 1-9.

11. Oh SJ, Kang J, Maeng I, Jin Suck S, Yong Min, et al. (2009) Nanoparticleenabled terahertz imaging for cancer diagnosis. Opt Express 17(5): 3469-3475.
12. Wilmink GJ, et al., (2010) Lasers Surg Med p. 42: 22.

13. Titova LV, et al., (2013) Intense $\mathrm{THz}$ pulses down-regulate genes associated with skin cancer and psoriasis: a new therapeutic avenue? Sci Rep 3: 2363.

14. Zhao S, Hu F, Xu X, Jiang M, Zhang W, et al. (2019) Electrically triggered dual-band tunable terahertz metamaterial band-pass filter based on Si3N4-V02-Si3N4 sandwich. Chinese Phys B 28(5): 048703.

15. Titova LV, Ayesheshim AK, Golubov A, Fogen D, Rodriguez Juarez R, et al. (2013) Intense THz pulses cause H2AX phosphorylation and activate DNA damage response in human skin tissue. Biomed Opt Express 4(4): 559-568.

16. Perera PGT, Appadoo DRT, Cheeseman S, Wandiynto JV, Linklater D, et al. (2019) PC 12 Pheochromocytoma Cell Response to Super High Frequency Terahertz Radiation from Synchrotron Source. Cancers 11(2): 162 .

17. Borovkova M, Serebriakova M, Fedorov V, Sedykh E, Vaks V, et al. (2017) Investigation of terahertz radiation influence on rat glial cells. Biomed Opt Express 8(1): 273-280.

18. Miyoshi N, Idehara T, Khutoryan E, Fukunaga Y, Bibin AB, et al. (2016) Combined Hyperthermia and Photodynamic Therapy Using a Sub-THz Gyrotron as a Radiation Source. J Infrared Milli Terahz Waves 37: 805814. 